Classification

Physics Abstracts

$68.55-61.50 \mathrm{~K}$

\title{
Investigation of Strain Relaxation Mechanisms in InGaAs/GaAs Single Layer Films
}

\author{
Filippo Romanato $\left({ }^{1}\right)$, Antonio Vittorio Drigo $\left({ }^{1}\right)$, Laura Francesio $\left({ }^{2}\right)$, Paolo Franzosi $\left({ }^{2}\right)$, \\ Laura Lazzarini $\left({ }^{2}\right)$, Giancarlo Salviati $\left({ }^{2}\right)$, Massimo Mazzer $\left({ }^{3}\right)$, Maria Rita $\operatorname{Bruni}\left({ }^{4}\right)$ and \\ Maria Grazia Simeone $\left({ }^{4}\right)$ \\ $\left({ }^{1}\right)$ Dipartimento di Fisica and Istituto Nazionale di Fisica della Materia (INFM), Università di \\ Padova, Via Marzolo 8, 35131 Padova, Italy \\ ${ }^{2}{ }^{3}$ Istituto CNR-MASPEC, via Chiavari 18/A, 43100 Parma, Italy \\ $\left({ }^{3}\right)$ Department of Materials, Imperial College, Prince Consort Road, London 3W72BP, U.K. \\ $\left({ }^{4}\right)$ C.N.R. I.C.M.A.T., Via Salaria km 29, 00015 Roma, Italy
}

(Received March 1; accepted June 27, 1995)

\begin{abstract}
In this work a strict comparison of the results obtained on InGaAs/GaAs heterostructures by HRXRD and RBS-channeling analysis shows a discrepancy in the In atomic fraction determined by the two techniques. The discrepancy leads to a difference in the reference lattice parameter of the relaxed film and, therefore, changes the description of the strain relaxation rate. After a discussion on the possible reasons for this discrepancy, the results have been interpreted as the influence of the atomic degrees of freedom internal to the lattice unit cell which could determinate the equilibrium shape of the unit cell. While it has been not strictly proved, the most reasonable hypothesis to explain the experimental results is that local ordering leads to a relaxed unit cell which is slightly tetragonal.
\end{abstract}

\section{Introduction}

In the last few years the investigation of the strain relaxation mechanisms in epitaxial heterostructures has led to the understanding of their possible applications [1-6]. In particular, the structural and opto-electronic characteristic modifications of pseudobinary non isovalent semiconductor compounds by means of the atomic bond strain condition have been described in theoretical works [7-13]. Moreover, strain can induce atomic displacements internal to the lattice unit cell that organize themselves in an ordered structure with long [14-16] or short [17] coherency length. Nevertheless, the experimental investigations of the influence of these internal degrees of freedom on the strain release processes are at the beginning. The aim of this work is to point out their role in the relaxation of $\mathrm{In}_{x} \mathrm{Ga}_{1-x}$ As single layer heterostructures MBE grown on GaAs substrates. In fact, in a previous work [18] on low (8-15\%) In concentration InGaAs/GaAs single layer heterostructures (SLH), we already pointed out some discrepancy between High Resolution X-Ray Diffraction (HRXRD) and Rutherford Backscattering Spectrometry (RBS) results. In this 
paper we extend the study of SLH to high (33\%) In concentration showing undoubtedly that the discrepancy between the In concentration as determined by XRXRD and RBS is confirmed.

This discrepancy cannot be ascribed to systematic errors and compels to a new and deeper elaboration of the physical quantities derived from HRXRD using the elastic continuum theory. As a consequence our results suggest a more precise examination of the unit cell deformation especially as far as its internal structure is concerned. To our knowledge our results are the first experimental evidence of the effectiveness of the unit cell structure of compound semiconductors in accommodating strain.

\section{Experimental}

The analyzed samples are SLH of $\operatorname{In}_{x} \mathrm{Ga}_{1-x}$ As grown at ICMAT in a conventional MBE system on [001] oriented Si doped GaAs substrates with an average dislocation density of $5.5 \times 10^{2} \mathrm{~cm}^{-2}$. The nominal value of the In atomic fraction is $x=33 \%$. The epilayer thickness ranges from $25 \mathrm{~nm}$ to $5 \mu \mathrm{m}$. A standard wet etching was used for substrate preparation in order to obtain a thin oxide film as passivation layer. Before the growth it was removed by heating at $580-600^{\circ} \mathrm{C}$ under arsenic flux. During the growth the substrate temperature was kept constant at $530^{\circ} \mathrm{C}$ for all the samples. The GaAs buffer and ternary layer growth rates were determined by RHEED oscillations.

RBS-channeling measurements were carried out at the Laboratori Nazionali di Legnaro by using a ${ }^{4} \mathrm{He}^{+}$beam. The chosen beam energies range from 2 to $3 \mathrm{MeV}$ according to the thickness of the sample. The scattering angle was set to $170^{\circ}$, but for the case of the thinner sample where it was set equal to $120^{\circ}$ to improve the thickness resolution. The beam charge collection was performed by using the whole scattering chamber as a Faraday cup reaching an accuracy better than $1 \%$. A high precision goniometric holder was used to perform the channeling analysis [19] and tetragonal distortion measurements [20].

The compositional depth profile of the samples can be measured from the relative height of the RBS signal corresponding to the different elements. In order to avoid any channeling effect that can affect the yield of the spectrum, during the acquisition the sample was tilted $5^{\circ}$ from [001] axis and azimuthally rotated. Taking in account the symmetry of the crystal, the sample was rotated of $45^{\circ}$ in step of $0.5^{\circ}$, beginning from the (110) planar channeling condition until the (100) plane was reached. At each angular step the same amount of charge was collected and the final spectrum thus resulted in an average of each angular position.

In our data reduction the experimental spectrum is simulated by a computer code through the use of a trial concentration profile until agreement with the experimental spectrum, within the statistics, is reached. An example of the results of this measurement and analysis procedure is shown in Figure 1, where the experimental and the computer simulated spectra of one of the samples are shown. With this procedure any systematic error is nearly completely compensated as discussed in reference [21]. The control on the beam charge allows us to accumulate a signal yield sufficient to decrease the relative statistic error below $1 \%$. The resulting precision of the $x$ determination results to be good and of the order of 0.3 at $\%$.

$\mathrm{X}$-ray measurements were performed at MASPEC on a high resolution $\mathrm{X}$ ray diffractometer using the symmetric 004 and the asymmetric 335 reflections. In order to obtain the parallel and perpendicular lattice mismatches, the measurements were performed both in the grazing incidence and the grazing emergence geometry. For each geometry four independent measurements were repeated after successive $90^{\circ}$ rotations around the surface normal.

To avoid the effect of the small $\left(<0.5^{\circ}\right)$ deviation of the physical surface from the nominal (001) crystallographic plane and of the layer lattice tilt with respect to the substrate caused by the dislocation network at the interface, the average values of the angular separation between the layer and substrate Bragg peaks after $180^{\circ}$ rotation were taken. In this way two independent 


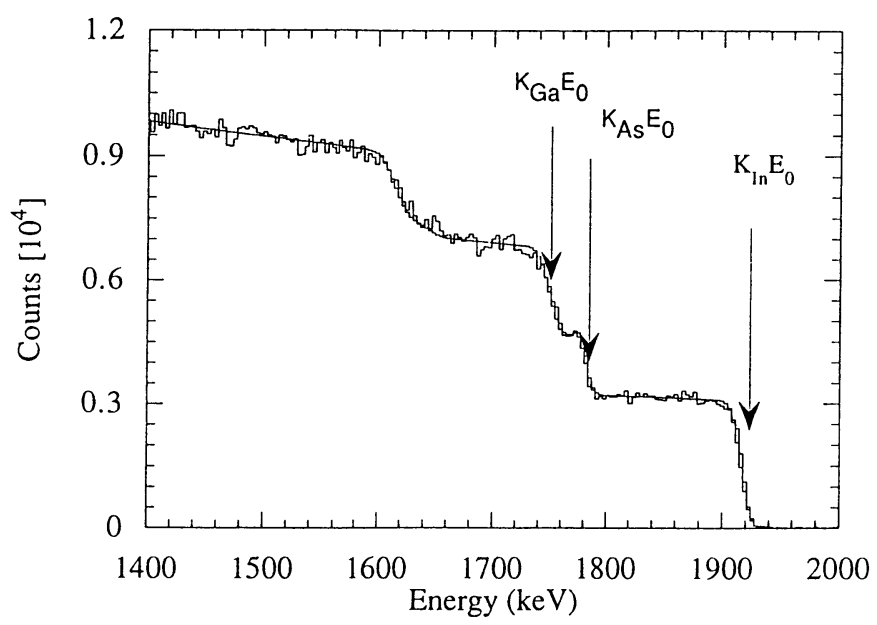

Fig. 1. $-2 \mathrm{MeV} \mathrm{He}^{+} \mathrm{RBS}$ experimental and computer simulated spectra of an InGaAs single layer grown on GaAs substrate. An excellent agreement between two spectra is achieved for an In composition $x=0.336$. The arrows show the energy, $\mathrm{KE}_{0}$, of $\mathrm{He}^{+}$ions backscattered at the surface from In, $\mathrm{Ga}$ and As atoms. The three $K$ represent the kinematics coefficients of the different elements and $E_{0}$ is the beam energy.

measurements of mismatch in the scattering planes corresponding to the $0^{\circ}-180^{\circ}$ and $90^{\circ}-270^{\circ}$ rotations were obtained. Finally, the mismatch values have been calculated from the measured values of the peak splitting [22].

\section{Results}

The main result of the HRXRD technique analysis is the measurement of the lattice parameter parallel, $a_{\|}$, and perpendicular, $a_{\perp}$, to the interface. In order to determine the deformation of the epilayer unit cell, the lattice parameters $a_{\perp}$ and $a_{\|}$must be compared with the value of the reference lattice parameter, $a_{x}$, of the completely relaxed InGaAs alloy. In fact, in the case of an epilayer grown on a [001] substrate with a biaxial strain field, the deformation can be described by the two equations

$$
\begin{gathered}
\varepsilon_{\perp}=\frac{a_{\perp}-a_{x}}{a_{x}} \\
\varepsilon_{\|}=\frac{a_{\|}-a_{x}}{a_{x}}
\end{gathered}
$$

that define the diagonal components of the strain tensor perpendicular and parallel to the interface, respectively.

In order to obtain $a_{x}$ by means of the HRXRD analysis, the following relationship between $\varepsilon_{\perp}$ and $\varepsilon_{\|}$must be used

$$
\varepsilon_{\perp}=-\frac{2 c_{12}}{c_{11}} \varepsilon_{\|}=-\alpha \varepsilon_{\|}
$$

where $c_{11}$ and $c_{12}$ are the InGaAs alloy elastic constants obtained by weighting the elastic constants of the end-point compounds, InAs and GaAs, with the In and Ga atomic fractions, respectively. 


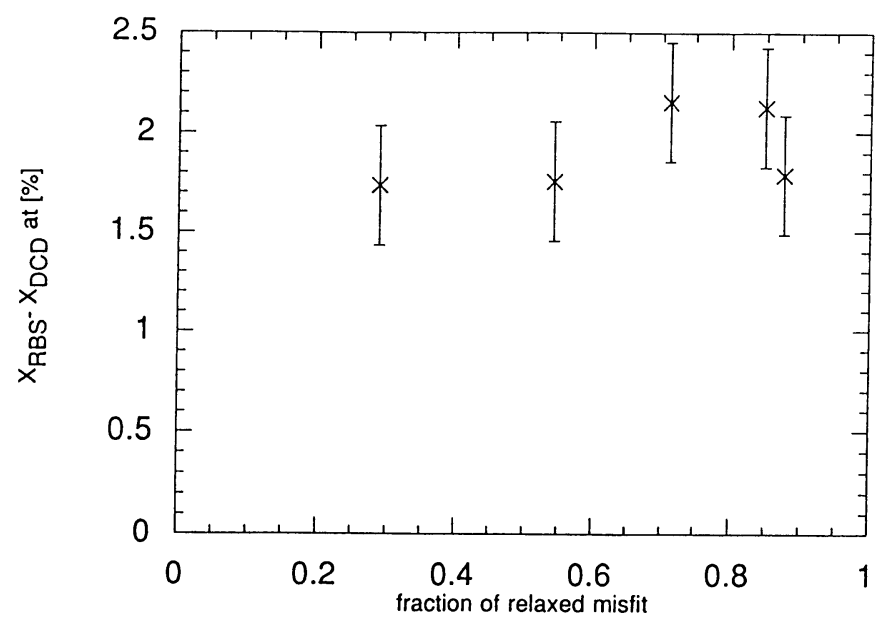

Fig. 2. - The difference between the In atomic fraction determined by RBS and HRXRD plotted as a function of the relative relaxed misfit, $\frac{f-\varepsilon_{\|}}{f}$.

It is worth noting that the elastic isotropic continuum theory, which equation (2) belongs to, can be applied to a crystal only if it has a tetragonally distorted cubic unit cell [23]. From equations (1a), (1b) and (2) the relaxed lattice parameter determined from HRXRD is given by

$$
a_{x_{\mathrm{HRXRD}}}=\frac{a_{\perp}+\alpha a_{\|}}{1+\alpha}
$$

Then the In atomic fraction, $x_{\mathrm{HRXRD}}$, can be obtained from the Vegard's law

$$
a_{x}=x a_{\mathrm{InAs}}+(1-x) a_{\mathrm{GaAs}}
$$

In Figure 2 the difference between the In atomic fraction determined by RBS, $x_{\mathrm{RBS}}$, and by HRXRD, is reported as a function of the relative relaxed misfit, $\frac{f-\varepsilon_{\|}}{f}$, where the misfit $f$ is defined as $f=\frac{a_{x}-a_{\mathrm{GaAs}}}{a_{\mathrm{GaAs}}}$. Figure 2 shows a nearly constant discrepancy of about 2 at $\%$ between the two measurements.

In Figure 3 the HRXRD measurements of $a_{\perp}, a_{\|}$, and the determinations of $a_{x_{\mathrm{RBS}}}$ and $a_{x_{\mathrm{HRXD}}}$ are reported as a function of the normalized relaxed misfit. The atomic fraction difference is reflected by Vegard's law in the difference between $a_{x_{\mathrm{RBS}}}$ and $a_{x_{\mathrm{HRXRD}}}$. Taking in account that the RBS analysis gives compositional measurements independent of the strain, we assume $a_{x_{\mathrm{RBS}}}$ as the correct lattice parameter of the unstrained $\operatorname{In}_{x} \mathrm{Ga}_{1-x}$ As alloy structure. It appears that $a_{\perp}$ relaxes towards $a_{x_{\mathrm{RBS}}}$ faster than $a_{\|}$. This result implies that the ratio between $\varepsilon_{\perp}$ and $\varepsilon_{\|}$are not constant all the misfit relaxation range long and, therefore, that equation (2) cannot be applied to the determination of $a_{x}$.

\section{Discussion}

In order to discuss the possible reason for the discrepancy shown in Figure 2 we must first analyze any possible systematic error in the used analysis techniques. Let us begin with RBS. 


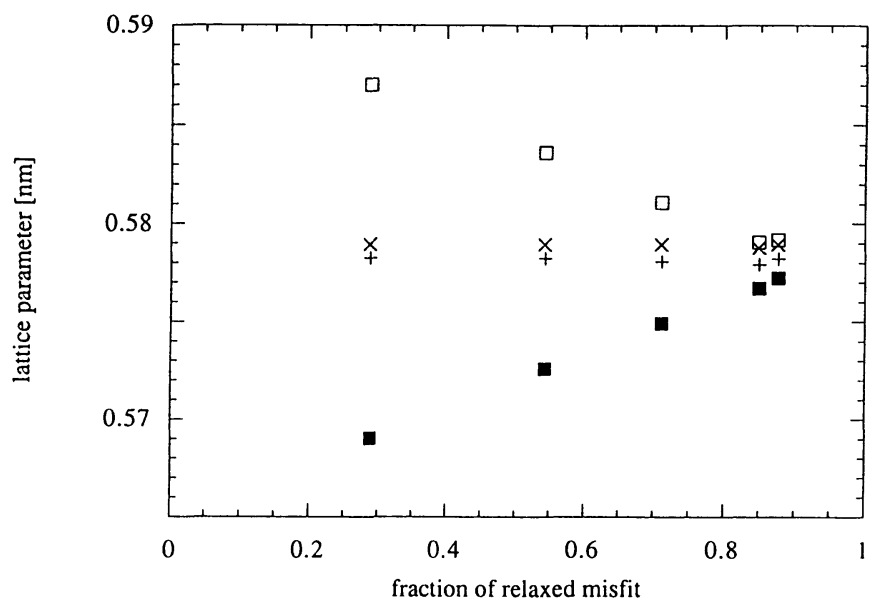

Fig. 3. - The lattice parameters $a_{\perp}, a_{\|}$determined by HRXRD (open and full square, respectively), and the relaxed lattice parameters determined by RBS, $a_{x_{\mathrm{RBS}}}(\times)$, and by HRXRD, $a_{x_{\mathrm{HRXR}}}(+)$, plotted as a function of the relative relaxed misfit, $\frac{f-\varepsilon_{\|}}{f}$. The error bar is comparable with the dimension of the symbols.

As it has been discussed in reference [21] the main source of error in the RBS analysis may come from the stopping power values used in the computer simulation of the spectra. We used the Ziegler's [24] compilation which is believed to be the most complete and accurate tabulation available so far. The maximum estimated uncertainty is below $10 \%$. Even though such an error were present, it would have a really negligible effect on the composition analysis because the same stopping power is used to analyze both the In signal and the GaAs signal of the epilayer. Moreover, the spectra of the end-point compounds have been perfectly simulated by using the appropriate stopping power and the Rutherford cross section.

As for X-ray diffraction, the used procedure is the best possible one and world-wide accepted. Moreover, it must be underlined that the ratio $a_{\perp} / a_{\|}$derived from HRXRD perfectly fits the values deduced from high precision channeling measurements [20,25].

After having excluded systematic errors of the experimental techniques we would like to address to the validity of the Vegard's law [26]. It has been deduced in the frame of the Virtual Crystal Approximation [27] (VCA) by means of X-ray diffraction experiments performed on completely relaxed alloy semiconductor samples. In particular, in the case of $\operatorname{In}_{x} \mathrm{Ga}_{1-x}$ As, despite the large misfit $\left(f=7.16 \%\right.$ ) between $a_{\mathrm{InAs}}$ and $a_{\mathrm{GaAs}}$ and on the contrary to the case of SiGe alloys [28], no deviation from the linear behaviour foreseen by Vegard's law has been measured [29]. In VCA model all the atoms are located on ideal lattices sites of the unit cell neglecting any possible difference of In-As and Ga-As length bond. As a consequence, Vegard's law gives the composition dependence of the atomic volume and is the right one to use in any description of the material that does not consider the internal structure of the unit cell and therefore, in our case, for the determination of the In atomic fraction.

Nevertheless, going to the internal description the alloy unit cell, EXAFS experiments have actually demonstrated [30-34] in different pseudobinary alloys of III-V and II-VI compounds that the distances between the different kind of anion-cation couples is not the same. In fact, the bond lengths are kept nearly equal to that of the end-point compounds, almost independently of the alloy composition, and only the angles between bonds change [35]. 
In reference [18] the validity of the elastic isotropic continuum approximation (Eq. (2)) has been already addressed to. Before entering this point we would like to stress that in reference [18] the discrepancy has been found as in the present samples, even though the growth and strain relaxation mechanisms are completely different. In fact, in the present case the high In concentration leads, initially, to a 3D growth mode with the formation of a high density of threading dislocations connected by short segments of misfit dislocations distributed over the epilayer-substrate interface. On the contrary, in our previous work the In concentration was lower than $15 \%$ and $2 \mathrm{D}$ growth mode leads to the formation of a misfit dislocation network at the interface.

All the other hypothesis having been excluded, we are left with the elastic continuum approximation which, as shown in Figure 3, seems to be no longer valid. This fact suggests to consider the internal structure of the unit cell. Mbaye et al. [36] showed that ternary semiconductors alloys, $\mathrm{A}_{x} \mathrm{~B}_{1-x} \mathrm{C}$, with precise value of $x(0.25,0.5,0.75)$ may crystallize in ordered structures where the different elements organize themselves in crystal sublattices. The same authors show that the misfit can be accommodated not only by the tetragonal distortion of the unit cell but very effectively by means of reciprocal movements of the monoelemental sublattices. Moreover, some theoretical works state that the equilibrium condition of these ordered structures does not necessarily correspond to the absence of tetragonal distortion [37].

Long range order can be revealed and investigated by X-ray diffraction, as it was shown for InGaP/GaAs layers $[38,39]$. The ordered structure in the cationic sublattice behaves as a natural $\mathrm{GaAs} / \mathrm{InAs}$ superlattice on $\{111\}$ planes, making allowed the $1 / 21 / 2 n / 2$ reflections, where $n$ is an integer. However, in the present sample no order has been observed, as demonstrated by the complete absence of $1 / 21 / 25 / 2$ reflections. Moreover, the RBS- HRXRD discrepancy sets in when strain relaxation begin to be effective. Nevertheless, the deformation mechanisms of the ordered structures should keep an effectiveness in disordered ternary alloys too [36]. In fact, the disordered alloy can be seen as the superposition of ordered clusters weighted according to the local composition and atomic arrangement. Mbaye et al. suggest the existence of an "epitaxial selection between different structures", driven by the strain, that should compel the atomic arrangement of the structure to choose the most efficient local order to minimize the enthalpy of the system.

Then the result of Figure 3 and of reference [18] can tentatively interpreted as follows. Before an important fraction of the initial misfit has been relaxed, the atomic internal degrees of freedom are frozen by the high stress of the epitaxial constrain that can be accommodated only by a tetragonal distortion of the unit cell; on the contrary, when a large part of the initial misfit has been relaxed, the structure approaches its equilibrium state with a substantial contribute of the internal atomic freedom degrees bringing the relaxed unit cell to an average tetragonal form. Moreover, the higher In concentration of the SLH described in this work with respect to the SLHs of reference [18] can explain the greater effectiveness of the internal degrees of freedom that should be greater the higher is the number of different bonds in the alloy (i.e. $x=50 \%$ ).

From an experimental point of view, a study with techniques probing the order of the atomic stereo correlation with a medium-short coherency length is necessary to deeper investigate the proposed interpretation. However the described results, if confirmed, represent the first evidence of the effectiveness of the internal atomic freedom degrees in strain relaxation.

\section{Conclusions}

In this work a strict comparison of the results obtained on InGaAs/GaAs heterostructures by HRXRD and RBS-channeling analysis shows a discrepancy in the In atomic fraction determined by the two techniques. The discrepancy leads to a difference in the reference lattice parameter of the relaxed film and, therefore, changes the description of the strain relaxation rate. After a 
discussion on the possible reasons for this discrepancy, the results have been interpreted as the influence of the atomic degrees of freedom internal to the lattice unit cell which could determinate the equilibrium shape of the unit cell. While it has been not strictly proved, the most reasonable hypothesis to explain the experimental results is that local ordering leads to a relaxed unit cell which is slightly tetragonal. This fact is important for two reasons: i) it can affect appreciably the strain relaxation rate; ii) the composition of ternary alloys as determined by HRXRD can be in error of as much as 2 at\%.

\section{References}

[1] Georgakilas A. and Christou A., J.Appl. Phys. 76 (1994) 7332.

[2] Molina S.I, Pacheco F.J. and Kidd P., Appl. Phys. Lett. 65 (1994) 2460.

[3] Chin T.P., Hou H.Q. and Tu C.W., Appl. Phys. Lett. 64 (1994) 2001.

[4] Chang S.Z., Lee S.C. and Shiao H.P., Appl. Phys. Lett. 64 (1994) 2417.

[5] Gosling T.J., Jain S.C. and Harker A.H., Phys. Status Solidi 146 (1994) 713.

[6] Gillard V.T., Nix W.D. and Freud L.B., J. Appl. Phys. 76 (1994) 7280.

[7] for a review see Zunger A., Wagner S. and Petroff P.M., J. Electr. Mat. 22 (1993) 3 and connected bibliography.

[8] Laks D.B., Wei S.H. and Zunger A., Phys. Rev. Lett. 69 (1992) 3766.

[9] Wei S.H. and Zunger A., Appl. Phys. Lett. 56 (1990) 662.

[10] Norman A.G., Seong T.S., Ferguson I.T., Booker G.R. and Joyce B.A., Semicon. Sci. Tech. 8 (1993) S9.

[11] Wei S.H. and Zunger A., Appl. Phys. Lett. 58 (1991) 2684.

[12] Chen A.C., Moy A.M., Pearah P.J., Hseieh K.C. and Cheng K.Y., Appl. Phys. Lett. 62 (1993) 1359.

[13] Cheng K.Y., Heish K.C. and Baillageon J.N., Appl. Phys. Lett. 60 (1992) 2892.

[14] Chu S.N.G., Logan R.A. and Tanbun-Ek T., J. Appl. Phys. 72 (1992) 4118.

[15] Onda T. and Ito R., Jpn. J. Appl. Phys. 28 (1989) 1544.

[16] Ni J., Lai X. and Gu B., J. Appl. Phys. 73 (1993) 4260.

[17] Ichimura M. and Sasaki A., J. Appl. Phys. 60 (1986) 3850.

[18] Mazzer M., Romanato F., Drigo A.V. and Carnera A., J. Cryst. Grow. 126 (1993) 125-132.

[19] Drigo A.V., Mazzer M. and Romanato F., Nucl. Instr. Meth. B63 (1992) 30-35.

[20] Carnera A. and Drigo A.V., Nucl. Instr. Meth. B44 (1990) 357.

[21] Armigliato A., Servidori M., Cembali F., Fabbri R., Rosa R., Corticelli F., Govoni D., Drigo A., Mazzer M., Romanato F., Frabboni S., Balboni R., Iyer S.S. and Guerrieri A., Microsc. Microanal. Microstruct. 3 (1992) 363-384.

[22] Salviati G., Lazzarini L., Ferrari C., Franzosi P., Romanato F., Berti M., Mazzer M., Drigo A.V., Bruni M.R., Simeoni M.G. and Gambacorti N., Scanning Microsc. 8 (1994) 943.

[23] Landau L.D. and Lifshitz E.M., Theory of Elasticity 3rd Ed. (Pergamon Press, New York).

[24] Ziegler J.F., Stopping Power and Ranges of Ions in Matter, Vol 4 (Pergamon Press, New York, 1977).

[25] Drigo A.V., Aydinly A., Carnera A., Genova F., Rigo C., Ferrari C., Franzosi P. and Salviati G., J. Appl. Phys. 66 (1989) 1975.

[26] Vegard L., Z. Phys. 5 (1921) 17.

[27] Norgheim L., Ann. Phys. (Leipz) 9 (1931) 607, 641.

[28] Dismukes J.P., Ekstrom L. and Paff R.J., J. Phys. Chem. 68 (1964) 3021.

[29] Wooley J.C. and Smith B.C., Proc. Phys. Soc. London 72 (1958) 241.

[30] Mikkelsen J.C. and Boyce J.B., Phys. Rev. B 28 (1983) 7130. 
[31] Oyanagi H., Takedo Y., Matsushita T., Ishiguro T., Yao T. and Sasaki A., Solid State Commun. 67 (1988) 453.

[32] Qteish A., Motta N. and Balzarotti A., Phys. Rev. B 30 (1989) 5987.

[33] Balzarotti A., Kitsiel A., Motta N., Zimnal-Starnawska M., Czyzyk M.T. and Podgorny M., Phys. Rev. B 30 (1984) 2295.

[34] Balzarotti A., Motta N., Kitsiel A., Zimnal-Starnawska M., Czyzyk M.T. and Podgorny M., Phys. Rev. B 31 (1985) 7526.

[35] Shabel M.C. and Martins J.L., Phys. Rev. B 43 (1991) 11873.

[36] Mbaye A.A., Wood D.M. and Zunger A., Phys. Rev. B 37 (1988) 3008.

[37] Yeh Chin-Yu, Chen A.B. and Sher A., Phys. Rev. B 43 (1991) 9138.

[38] Liu Q., Lakner H., Scheffer F., Linder A. and Prost W., J. Appl. Phys. 73 (1993) 2770.

[39] Francesio L., Franzosi P., Caldironi M., Vitali L., Dellagiovanna M., Di Paola A., Vidimari F. and Pellegrino S., Mater. Sci. Engng. B28 (1994) 219. 